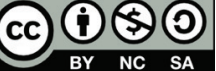

Esta obra está bajo una Licencia Creative Commons

Atribución-NoComercial-Compartirlgual 4.0 Internaciona

Talleres de educación ambiental: sistematización de experiencias en el marco de un proyecto interinstitucional de extensión universitario

Eleana Spavento

Trayectorias Universitarias, 6(11), e044, 2020

ISSN 2469-0090 | https://doi.org/10.24215/24690090e044

https://revistas.unlp.edu.ar/TrayectoriasUniversitarias

Universidad Nacional de La Plata

La Plata | Buenos Aires | Argentina

\section{Talleres de educación ambiental: sistematización de experiencias en el marco de un proyecto interinstitucional de extensión universitario}

Environmental education workshops: systematization of experiences within the framework of an interinstitutional project of university extension

\section{Eleana Spavento}

https://orcid.org/0000-0002-3810-8952

eleana.spavento@agro.unlp.edu.ar

Facultad de Ciencias Agrarias y Forestales

Laboratorio de Investigaciones en Maderas

(LIMAD) | Universidad Nacional de La Plata

Camila Mateo

https://orcid.org/0000-0002-0871-0440

cronopiosp@gmail.com

Facultad de Ciencias Agrarias y Forestales |

Universidad Nacional de La Plata | Argentina

\author{
María Mercedes Refort \\ https://orcid.org/0000-0001-7942-3492 \\ mmrefort@gmail.com \\ Facultad de Ciencias Agrarias y Forestales \\ Laboratorio de Investigaciones en Maderas \\ (LIMAD) | Universidad Nacional de La Plata \\ María Fernanda Rossi Batiz \\ https://orcid.org/0000-0003-1239-2729 \\ mfrb@fcnym.unlp.edu.ar \\ Facultad de Ciencias Naturales y Museo I \\ Universidad Nacional de La Plata | Argentina
}

\title{
RESUMEN
}

Este trabajo tiene como objetivo sistematizar experiencias enmarcadas en el proyecto interinstitucional de extensión: "Redescubriendo los Humedales de Berisso: Talleres de Educación Ambiental”, de la Universidad Nacional de La Plata, durante el período 2017-2019. Dicha experiencia consistió en la aplicación de una estrategia de enseñanza, modalidad taller, planteada a fin de fomentar la actitud crítica, participativa y responsable en estudiantes de Escuelas de Educación Primaria, frente a la situación ambiental de los humedales. Asimismo, se planteó el fortalecimiento "Universidad-Sociedad" en torno a la reflexión sobre la importancia y conservación de dicho ecosistema. La descripción de la experiencia se estructuró en tres momentos: "antes": pensar el proyecto, "durante": desarrollo de los talleres y "después": reconstrucción del proceso vivido. Del análisis reflexivo surge que los talleres permitieron a los estudiantes el trabajo teórico-práctico integrado, dinámico y colaborativo, logrando la deconstrucción-reconstrucción de sus saberes curriculares y experienciales. Además, se potenció la interdisciplinariedad de saberes y la interacción estudiantes-docentes. En general, esta estrategia de enseñanza fundamentada en los humedales de Berisso, permitió configurar el saber pedagógico desde el pensamiento y la reflexión del "mundo real", reconociendo e incorporando como parte del proceso, al contexto social.

\section{ABSTRACT}

This work aims to systematizeof an experience within the framework of the interinstitutionalproject of university extension "Rediscovering the Wetlands of Berisso-Environmental education workshop" of the National University of La Plata, during 2017-2019 periods. This experience consisted of a teaching strategyimplementation, workshop modality, proposed in order to promote the critical, collaborative and responsibility attitude in primary school students in according to this wetlands environmental situation. Also, the "University-Society" strengthening about wetlands importance and conservation reflexion was considered. The experience description was structured in three moments: "before": think about the project, "during": workshops development and "after": lived process reconstruction. From thoughtful analysis it emerges that the workshops allowed students to work integrally, dynamically and collaborativelyachieving deconstruction-reconstruction of them curricular and experienceal knowledge. In addition, the interdisciplinarity of knowledge and student-teacher interaction was enhanced. In general, this teaching strategy based on the Berisso wetlands, allows shaping pedagogical knowledge from the "real world" thought and reflection, recognizing and incorporating the social context within the process.

\section{PALABRAS CLAVE}

humedales,

escuelas de educación primaria, Universidad Nacional de La Plata.
KEY WORDS

wetlands,

primary education schools, National University of La Plata. 


\section{CONTEXTUALIZACIÓN}

Este trabajo contempla talleres realizados (período 2017-2019) en el marco del proyecto interinstitucional de Extensión Universitaria: "Redescubriendo los Humedales de Berisso: Talleres de Educación Ambiental" y tiene como objetivo describir, analizar e interpretar críticamente -sistematizar- la experiencia interdisciplinaria centrada en talleres desarrollados en Escuelas de Educación Primaria (EEP), a fin de entender si los mismos, responden al objetivo general del proyecto y a la demanda socio-ambiental de la región.

La interdisciplinariedad estuvo dada por la vinculación de diferentes Unidades Académicas (UA) de la Universidad Nacional de La Plata (UNLP). Los actores-promotores directos del cambio estuvieron representados por docentes-extensionistas y estudiantes de las siguientes UA: Facultad de Ciencias Naturales y Museo (FCNyM), en carácter unidad ejecutora del proyecto y Facultades de Ciencias Agrarias y Forestales (FCAyF), Ciencias Médicas (FCM) y de Artes (FA) en carácter de participantes. Los actores-motores indirectos de motivación (Cabo 2000, tomado de Pérez de Maza, 2016) estuvieron representados por docente y alumnos (5-9 años) de diferentes EEP.

El contexto temporal de la experiencia se sitúa a partir del año 2016, momento en el que se planteó y diseñó el primer plan. No obstante, aquí focalizaremos en los talleres desarrollados durante el período especificado.

Para su análisis reflexivo y reinterpretación se considerarán los re- 
gistros de información aportados por los actores antes mencionados y se contemplará en la narración, la vinculación dialógica entre la reflexión crítica de la experiencia previa, los actores directos e indirectos, su interacción social-comunicación y su "hacer" (Edelstein, 2000). Para ello se asumió que las prácticas se desarrollan en diferentes contextos sociales, culturales, históricos e institucionales, adquiriendo una significación para la sociedad y los propios docentes (Achilli, 1986), por lo que los roles de los protagonistas debieron readaptarse continuamente a fin de propiciar el aprendizaje.

Desde la UNLP, se reconocen tres funciones fundamentales de desarrollo y fomento de conocimientos: la enseñanza, la investigación y la extensión. La primera, procura generar un contacto directo entre quienes participan de la misma, desarrollando la aptitud de observar, analizar y razonar, persiguiendo que los estudiantes y docentes tengan juicio propio, espíritu crítico, curiosidad científica, iniciativa y responsabilidad. La segunda, procura desarrollarse fomentando la investigación básica, humanística, artística y aplicada, así como el desarrollo, la innovación y la vinculación tecnológica, definiendo áreas prioritarias en base a sus objetivos, volcando sus recursos principalmente procurando alcanzar la excelencia, pertinencia y calidad. La tercera, persigue contribuir a la búsqueda de respuestas a problemas sociales, fundamentalmente de aquellos sectores más vulnerables por no tener sus derechos esenciales garantizados. Por lo tanto, la extensión universitaria constituye el principal medio de la UNLP para lograr su función social, contribuyendo al tratamiento de los problemas que afectan al bienestar de la comunidad, la reconstrucción del tejido social, el desarrollo económico sustentable y el fortalecimiento de la identidad cultural (Estatuto UNLP, 2008).

En este sentido, deben considerarse propósitos, contextos, métodos didácticos y criterios de acción ya que no hay metodología, sin tratamiento del contenido y sin entender la problemática del sujeto que aprende (Edelstein,1996). En relación con ello, Edwards (1985) expresa la idea de que la forma también es contenido; forma en que se conjugan diferentes dimensiones del conocimiento: de transmisión, de repetición del contenido, de textualidad de respuesta, de dinámica en la interrogación y de interacción entre lo explícito e implícito.

En este contexto, el objetivo del proyecto se concentra en fomentar una actitud crítica, participativa y responsable de la comunidad en relación a la situación de los humedales, a partir de la articulación de establecimientos educativos-comunidad. Como así también, en propiciar un espacio de intercambio de saberes locales y conocimiento académico, tendientes a mejorar y sostener el buen estado ambiental de los humedales y comprender su rol frente a las adversidades naturales, principalmente, inundaciones. 
En este contexto, el objetivo del proyecto se concentra en fomentar una actitud crítica, participativa y responsable de la comunidad en relación a la situación de los humedales, a partir de la articulación de establecimientos educativos-comunidad. Como así también, en propiciar un espacio de intercambio de saberes locales y conocimiento académico, tendientes a mejorar y sostener el buen estado ambiental de los humedales y comprender su rol frente a las adversidades naturales, principalmente, inundaciones.

Para dar respuesta a estos objetivos, entre otras actividades, se contempla:

Realización de talleres de conceptualización y discusión sobre los humedales: contaminación, sustentabilidad y conservación.

Realización de talleres de actividades manuales (maquetas, reconstrucciones, afiches, dibujos) y lúdicas, relacionadas con la temática.

Esos talleres abordan las temáticas ambientales-biológicas más importantes que atraviesan a los humedales, específicamente a los de Berisso.

\section{DESCRIPCIÓN DE LA EXPERIENCIA}

Para el desarrollo de este trabajo, la experiencia fue estructurada en tres momentos: "antes": pensar el proyecto, "durante": desarrollo de los talleres y "después": reconstrucción del proceso vivido. Finalmente, se realizó un análisis integral de la propuesta.

\section{Pensar el proyecto: el antes}

El proyecto surgió a partir de observaciones e interrogantes en el contexto académico integral de docentes, investigadores y estudiantes de grado de la FCNyM, como parte de sus investigaciones. En base a ello, se vislumbraba que el ecosistema del humedal local no tenía buena sanidad y sus servicios ecosistémicos no estaban funcionando como resultado de la contaminación y el avance antrópico, por lo que se decidió actuar en consecuencia y a partir de ello, se diseñó el plan. Cabe destacar que a diferencia de lo que ocurre actualmente, en ese período no existía una demanda social concreta en torno a estos ecosistemas, a la cual responder desde la UNLP. 


\section{Desarrollo de los talleres: el "durante"}

Esta etapa contempla el momento de realización de los talleres. Los mismos se desarrollaron en diferentes EEP y tuvieron una duración variable, acorde a sus disponibilidades horarias y/o solicitudes. Si bien, como en toda etapa, hubo una fase inicial de organización de tareas-responsabilidades y vinculaciones con las EEP, en esta instancia nos focalizaremos en el contexto áulico de las EEP.

Los talleres comenzaban con una presentación general del proyecto y desarrollo de los contenidos básicos referentes al humedal (definición RAMSAR (1971), importancia, conservación, grupos biológicos (mamíferos, artrópodos, plantas, etc.), entre otros. Conjuntamente se trabajaba con instancias prácticas divididas por grupos biológicos a cargo de 2-3 representantes de diferentes UA (UNLP) cada uno. Para ello, se utilizaba material (de herbario, entomológico, etc.) y se realizaban actividades diversas (actividades lúdicas, para completar, etc.), Figura 1, 2.El material creado, era recogido por los docentes-extensionistas y actualmente constituyen, parte de la memoria del proyecto y del registro de información interpretado en este trabajo.

Se optó por esta vinculación teórico-práctica a fin de trabajar participativa- colaborativamente, otorgándoles "empoderamiento" a los estudiantes para que sean ellos los generadores de interrogantes y formadores de contenidos durante el proceso de aprendizaje. Consecuentemente con lo expresado por Zúñiga, se pretendió "fortalecer colectivos, identidades, empoderar a los sujetos, hacerlos partícipes en la toma de decisiones y en la construcción de su propia historia" (1995, tomado de Jara Holliday, 2012, p. 159). Este es un aspecto muy importante dentro del contexto social en el que están enmarcados los humedales ya que muchos de los participantes de los talleres, se vinculan muy estrechamente a los mismos, con actividades que van desde la conservación hasta la recreación. En todo momento se intentaba trabajar de manera multilateral y colaborativa, evitando la concepción unilateral de educación (Freire, 1997). De este modo, se intentaba promover el aprendizaje autodidacta y exploratorio. Además, se buscaba enseñar basándose en la inteligencia cultural, cambiando su significado y creando un nuevo conocimiento (Flecha, 2004); se dirigía a que los estudiantes relacionaran, compararan y establecieran nexos a partir de lo dado para recuperar lo sabido, sea de un conocimiento curricular y/o natural o experiencial, y formaran "puentes" con el nuevo conocimiento, en torno a los humedales. Esto se constituía luego, en el punto de partida de otras propuesta o modificaciones didácticas (Litwin, 1997).

El cierre del encuentro-taller se realizaba recogiendo las opiniones de los participantes de las EEP, las cuales sirvieron de registro de información y de base para replantear talleres/proyectos posteriores. 


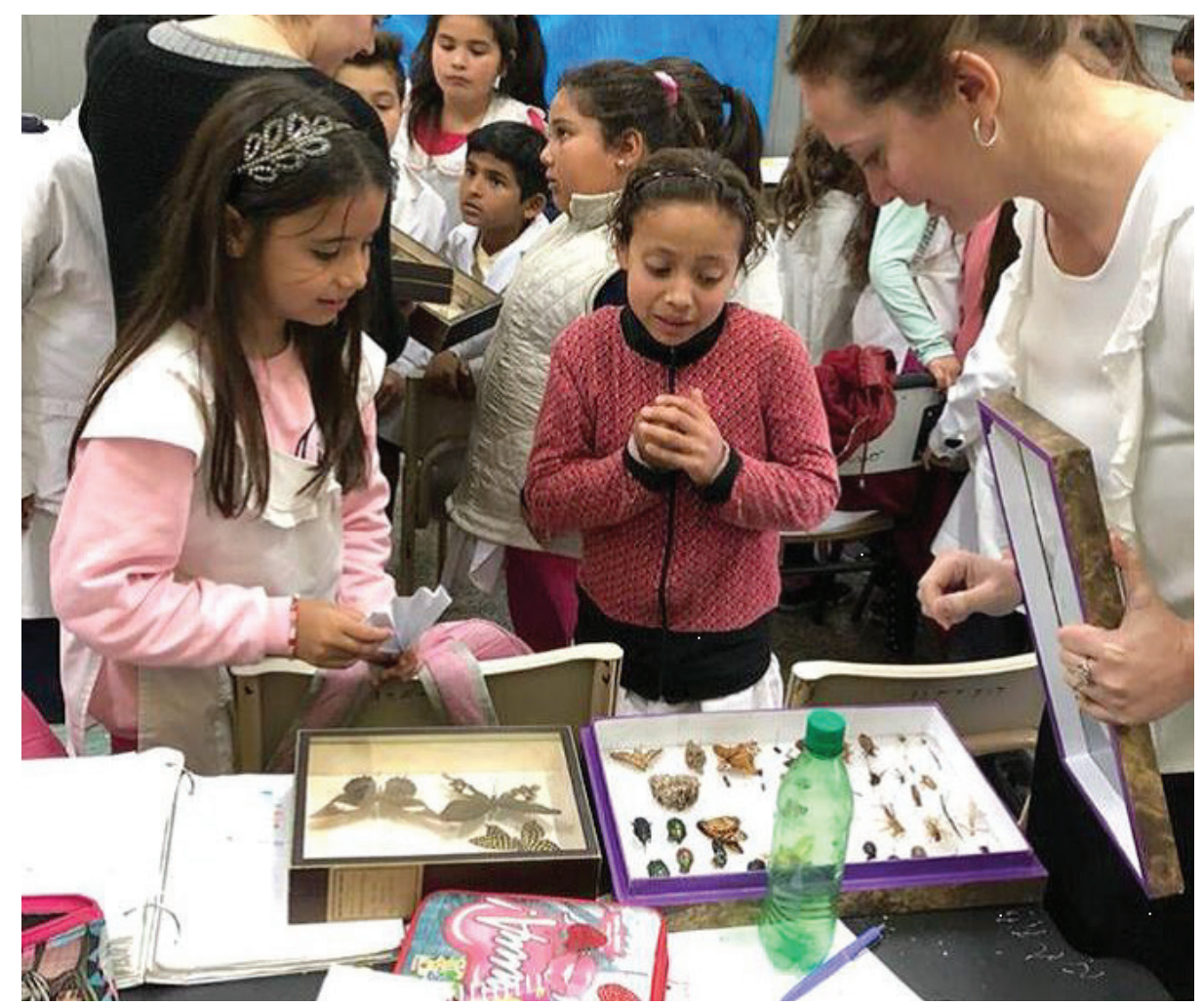

Figura 1: material entomológico, 2019, Ma. Fernanda RossiBatiz.

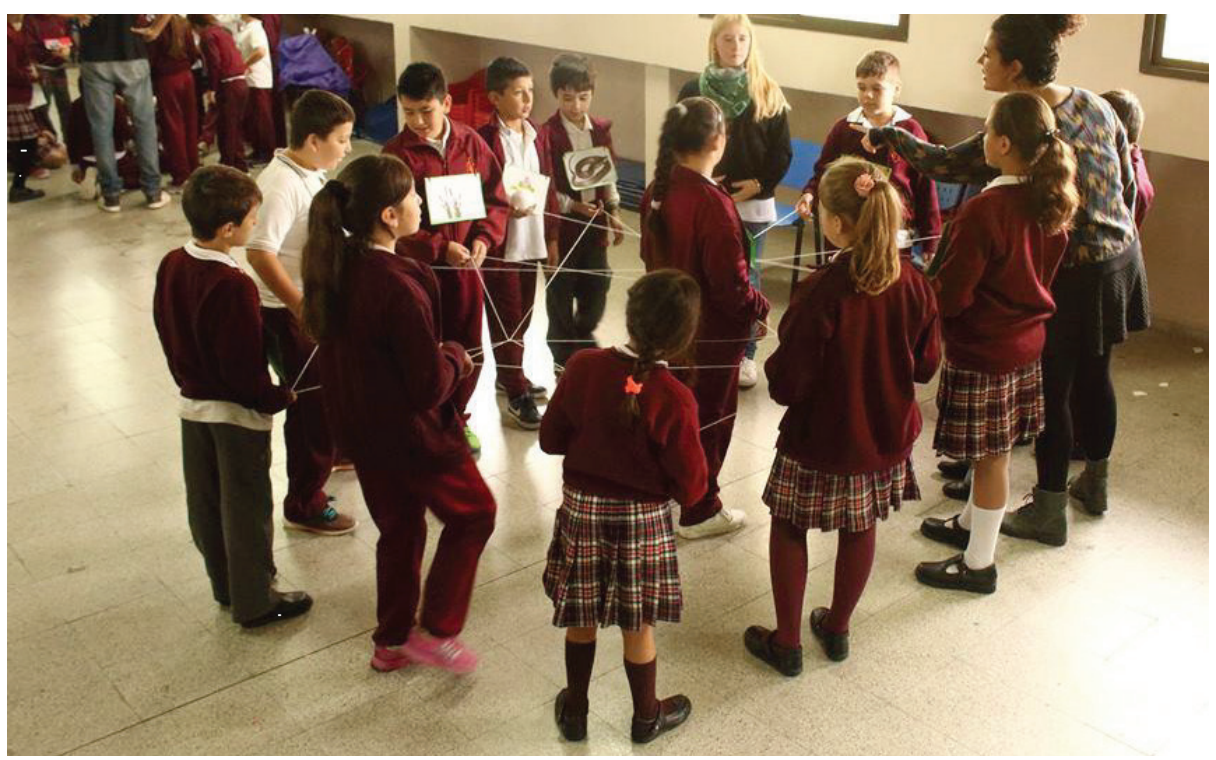

Figura 2: actividades lúdicas, 2017, Ma. Fernanda RossiBatiz.

\section{Reconstrucción del proceso vivido: el después}

Culminado el desarrollo de cada taller se realizaban reuniones entre los integrantes del proyecto y se comenzaba el proceso de repensar lo realizado, analizar las vivencias y las percepciones de cada experiencia; se analizaba el proceso, los aciertos y desaciertos, se retomaban las voces de los docentes-extensionistas que habían llevado a cabo la coordinación-organización de los talleres, como así también la de los alumnos y docentes de las EEP (registros de información). 


\section{PROCESO DE SISTEMATIZACIÓN}

Un proceso de sistematización es entendido como una interpretación crítica de experiencias a partir de su ordenamiento y reconstrucción, descubriendo o explicitando la lógica del proceso vivido, los factores que han intervenido en dicho proceso, cómo se han relacionado entre sí, y por qué se han hecho de ese modo (Jara Holliday, 2018, 2003). Por lo que, esta sistematización refiere a una experiencia parcial y en retrospectiva, dado que abarca un período (2017-2019) y es analizada, finalizada la misma por lo que derivó de una intervención intencionada-participativa, involucrando a quienes la vivenciamos, como informantes clave y como actores reflexivos, proponiendo cursos de acción, es decir, siendo protagonistas.

\section{Dimensiones de la experiencia}

De acuerdo con lo detallado anteriormente, el objeto a sistematizar queda constituido por los talleres de educación ambiental, como modalidad de trabajo durante el período 2017-2019.

Mientras que el eje de sistematización, es metodológico y consiste en el análisis de la modalidad de trabajo para la educación ambiental, brindada desde la Universidad (UNLP).

\section{Registro de información}

Uno de los aspectos relevantes al sistematizar una experiencia, es disponer de registros que documenten las acciones, intencionales o no, para utilizarlos como fuente de información que nos permita decodificarla; constituyen los "testigos" o "rastros del pasado", el diagnóstico previo o "perfil de entrada" que permite reconstruir la memoria de la experiencia (Barragán Cordero \& Torres Carrillo, 2017; Jara Holliday, 1994; 2018).

En esta experiencia se trabajó con un registro inicial, constituido por reportes obtenidos en las reuniones de los integrantes del proyecto e incluso, por los propios planes presentados, como así también por demandas sociales (en este trabajo, representado por las EEP) recibidas con el transcurrir del proyecto.

Seguidamente, la unidad de análisis estuvo constituida por los estudiantes y su relación con la experiencia, cuya unidad de muestreo estuvo constituida en el contexto áulico; el registro se constituyó en los trabajos prácticos realizados por los alumnos y en las conversaciones registradas a través de anotaciones durante el encuentro-taller. Se trabajó con muestreo total de la población (Mendieta Izquierdo, 2015).

Por último, la unidad de análisis estuvo centrada nuevamente en los docentes integrantes del proyecto a través del registro de reuniones realizadas, como así también con los docentes de las EEP o bien, 
a través de sus comentarios a posteriori del desarrollo del taller (quienes en muchos casos, demandaban la repetición de los encuentros o bien, alguna cuestión ambiental específica). A través de estos registros se intentó revelar la importancia de los talleres a fin de conocer si los mismos responden al objetivo general del proyecto de extensión y a la demanda socio-ambiental. Para ello se retomaron las decisiones, valoraciones, cambios, marchas y contramarchas de la propuesta, analizando la evolución de la implementación y el planteamiento de futuras intervenciones y/o reestructuración de la propuesta.

Del análisis de todos los registros se constituyó una reconstrucción crítica reflexiva que permitió establecer nuevas propuestas de mejoras.

\section{Reconstrucción histórica}

En la Figura 3 se resume, mediante una infografía, la reconstrucción histórica de todo el proceso de sistematización.

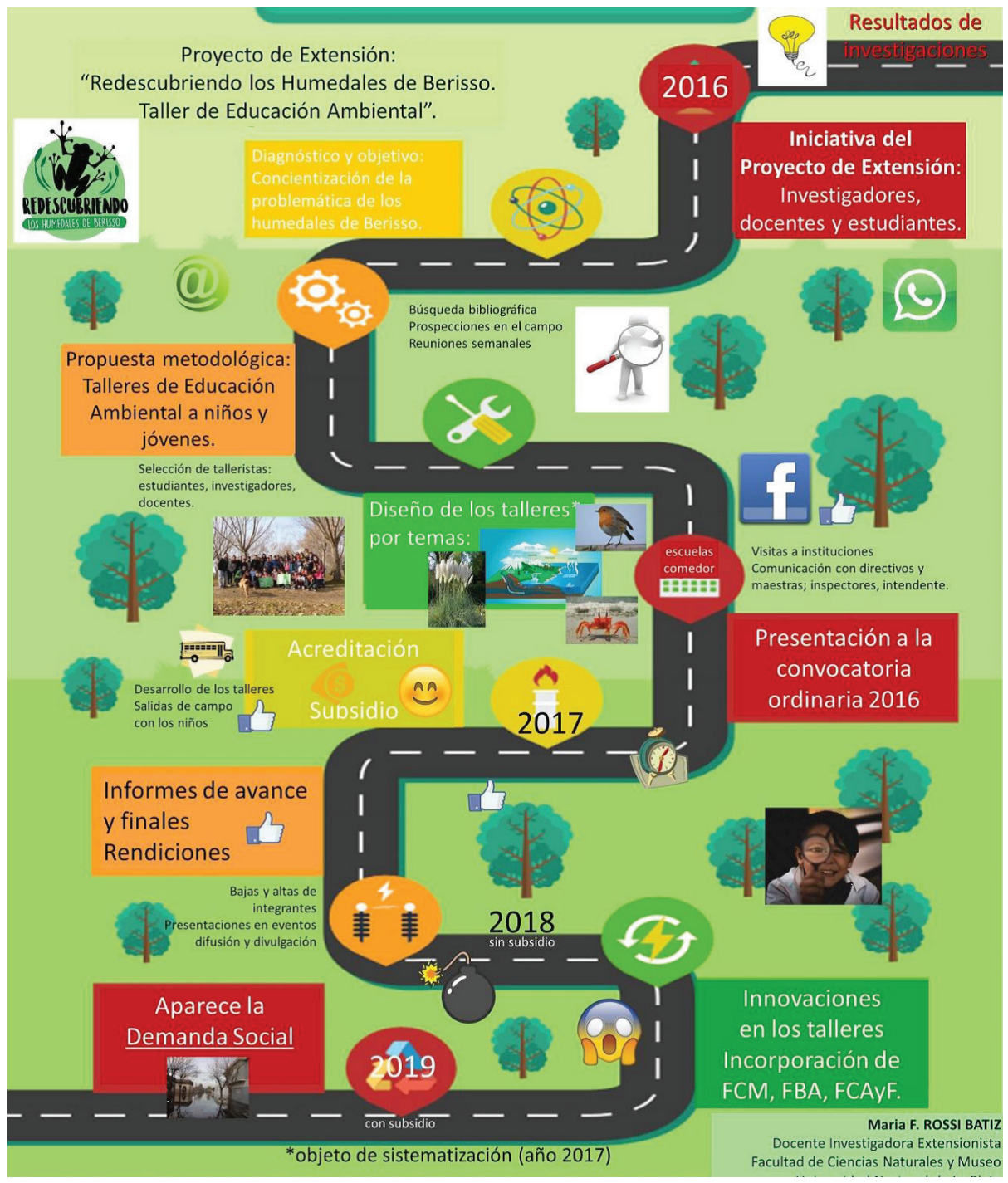


De acuerdo con lo esquematizado (Figura 3), durante los tres momentos considerados en este trabajo (antes, durante, después), se fueron planteando estrategias, muchas de ellas mediadas por tecnologías, que fueron conduciendo a otras estrategias para llevar adelante los objetivos del proyecto, entre ellas, los talleres de vinculación curricular interdisciplinaria en los cuales se fundamenta este trabajo.

\section{CONCLUSIONES}

En términos generales, los talleres constituyen una experiencia formativa, donde se ponen en juego saberes técnicos curriculares con saberes experienciales. En nuestro caso, permitieron que los estudiantes trabajaran integrada y colaborativamente, deconstruyendo y reconstruyendo los conocimientos curriculares y experienciales, contextualizándolos a los humedales de Berisso, construyendo además, nuevos vínculos. En este sentido, se transformó la relación de estudiantes como "depósito de contenido" y de docentes como "depositadores de contenidos", por una relación colaborativa donde adoptaron formas activas y desestructuradas de generación de sus propios conocimientos; se transformó incluso, la relación estudiante-estudiante a través de intercambios de pareceres (propios de sus vivencias) que potenciaban el desarrollo de la actividad. Por lo que, la forma de abordar los contenidos permitió otros contenidos como la interdisciplinariedad, la integración teórico-práctico, la dinámica en el desarrollo de la actividad y la interacción estudiante-docente y estudiante-estudiante.

Es por ello que a partir del aprendizaje internalizado a través de estos talleres, en consonancia con lo desarrollado a través de estas líneas, este ejercicio crítico y reflexivo, en el cual los docentes nos asumimos como intelectuales transformadores de la realidad (Giroux, 1990), nos ha interpelado, motivado y permitido hallar aspectos, relaciones, sentimientos y voces que no habíamos percibido hasta tanto realizar este análisis.

Además nos permitió reflexionar de manera continua y configurar-reconfigurar el saber pedagógico desde el pensamiento y la reflexión de lo acontecido en el "mundo real". En este sentido, desde el proyecto se reconoce que la construcción participativa se da y potencia cuando se reconocen e incluyen los actores sociales en el acto formativo. Y es a través de este camino que consideramos debemos continuar, afianzando la reconstrucción personal y colectiva de los talleres y retroalimentando la experiencia misma. 


\section{BIBLIOGRAFIA}

Achilli, E. (1986). “La práctica docente: una interpretación desde los saberes del maestro". Cuadernos de Formación Docente, 1(2). Universidad Nacional de Rosario.

Barragán Cordero, D. \& Torres Carrillo, A. (2017). “La sistematización como investigación interpretativa crítica". Bogotá, Colombia. Editorial El Búho Corporación síntesis.

Edelstein, G. (2000). “El análisis didáctico de las prácticas de la enseñanza. Una referencia disciplinar para la reflexión crítica". Revista del Instituto de Investigaciones en Ciencias de la Educación, Año IX:17, 7 pp.

Edelstein, G. (1996). “Un capítulo pendiente: el método en el debate didáctico contemporáneo". En A. Camillonietal. Corrientes didácticas contemporáneas (pp. 75-90). Buenos Aires. Ed. Paidós

Edwards, V. (1985). "La relación de los sujetos con el conocimiento". Parte integral de la tesis de Maestría vinculada al Programa Interdisciplinario de Investigación en Educación, PIIE. DIIE. México.

Estatuto UNLP. (2008). Universidad Nacional de La Plata. Disponible en: https:// unlp.edu.ar/frontend/media/20/120/722e7f1 b616ac158e02d148aaeb762aa.pdf

Flecha, R. (2004). "La Pedagogía de la Autonomía de Freire y la Educación Democrática de personas adultas". Revista Interuniversitaria de Formación del Profesorado, 18(2), 27-43. [Fecha de Consulta 17 de Enero de 2020]. ISSN:02138646. Disponible en: https://www.redalyc.org/articulo.oa?id=274/27418203

Freire, P. (1997). "Pedagogía de la autonomía. Saberes necesarios para la práctica educativa". México-España. Ed. Siglo veintiuno s.a.

Giroux, H. (1990). “Los profesores como intelectuales. Hacia una pedagogía crítica del aprendizaje". Barcelona, España. Ed. Paidós.

Jara Holliday, O. (2018). "La sistematización de experiencias: práctica y teoría para otros mundos posibles. Colombia. Centro Internacional de Educación y Desarrollo Humano. $1^{\text {a }}$ edición.

Jara Holliday, O. (2012). “La sistematización de experiencias práctica y teoría de otros mundos posibles" [Extractos]. Centro de Estudios y Publicaciones Alforja. CEAAL San José, Costa Rica. Ed. IntermónOxfam.

Jara Holliday, O. (2003). "Para sistematizar experiencias". Revista del equipo de Innovaciones Educativas DINESST-MED20, 10 pp. 
rica y práctica". Costa Rica. 1ra Edición, Centro de Estudios y Publicaciones, Alforja.

Litwin, E. (1997). “Las configuraciones didácticas. Una nueva agenda para la enseñanza superior”. Buenos Aires-Barcelona-México. Ed. Paidós.

Mendieta Izquierdo, G. (2015). "Informantes y muestreo en investigación cualitativa". Revista Investigaciones Andina, 17(30), 1148-1150.

Pérez de Maza, T. (2016). "Sistematización de experiencias en contextos universitarios". Guía didáctica". Universidad Nacional Abierta. Subprograma de Extensión Universitaria. Ediciones del Vicerrectorado Académico. Venezuela. 1ra Edición.

RAMSAR (1971). “QQué son los humedales?" Documento informativo Ramsar No. 1. Disponible en: https://www.ramsar.org/sites/default/files/documents/library/info2007sp-01.pdf 